\title{
A New Approach to the Problem of Motion in General Relativity
}

\author{
ENRICO MASSA * \\ Istituto di Matematica dell'Università di Genova
}

Received November 4, 1970

\begin{abstract}
The dynamical meaning of the equations $T^{i j} \|_{j}=0$ is derived as a consequence of the mathematical structure of Einstein's equations. A generalization of Lichnerowicz's analysis of the gravitational equations is proposed.
\end{abstract}

\section{$\S 1$. Introduction}

In this paper we discuss the problem of motion for a material continuum in the framework of the general theory of relativity ${ }^{1}$.

On this subject there is already a very extensive literature $[1 \div 12]$. The aim of the present contribution is to show that the general structure of the problem is intrinsically very simple.

To avoid unnecessary complications, we shall restrict our analysis to those systems whose four-velocity field $V^{i}$ and density $\mu$ are expressed in terms of the energy-momentum tensor $T_{i j}$ by the eigenvalue equation [13]

with the normalization

$$
\left(T_{i j}+\mu g_{i j}\right) V^{j}=0
$$

$$
g_{i j} V^{i} V^{j}=-1 \text {. }
$$

We prove that if we assume the validity of Einstein's equations

$$
G_{i j}=-k T_{i j}
$$

and impose certain consistency conditions for the initial and boundary data of the gravitational problem, Eqs. (1.1), (1.2) are mathematically equivalent to the divergence equations

$$
T^{i j}=0 \text {. }
$$

* Lavoro eseguito nel centro di Matematica e Fisica Teorica del C.N.R. presso l'Università di Genova.

1 Throughout the paper, Latin indices will run from 1 to 4 , and Greek indices from 1 to 3 , unless otherwise stated. The metric is assumed to be of normal hyperbolic type with signature $(++t-)$.

Partial derivatives will usually be indicated by a comma; covariant derivatives by a double vertical stroke (like in Eq. (1.4)). 
We then indicate how this fact may be regarded as a general proof that Eqs. (1.4) are the equations of motion for the given material continuum.

This result, although obtained under the simplifying assumption (1.1), may be easily extended to more general cases, e.g. to the case when an electromagnetic field is present.

However, we shall not discuss this generalization here.

In Section 2 we analyse the mathematical structure of Eqs. (1.3), and prove the validity of several Lichnerowicz-type decompositions of these equations under very weak conditions. The main results are stated at the beginning of Section 2, and proved in Lemma 2.1 and Lemma 2.2.

In Section 3 we discuss the relativistic formulation of the problem of motion, and use the material of Section 2 to obtain the desired conclusions.

Finally, in the Appendix, we indicate a general uniqueness theorem for symmetric hyperbolic systems of partial differential equations.

This theorem is not very original - in fact, it is borrowed from ref. [14] and adapted to the problem in study - and is reproduced here for convenience of the reader.

\section{§ 2. Mathematical Preliminaries}

In this section we prove a general result concerning the mathematical structure of Einstein's equations in the mixed initial and boundary value problem.

In the space-time manifold $\mathscr{V}_{4}$, let $\Gamma$ be a $\left(C_{1}, \tilde{C}_{2}\right)^{2}$ congruence of time-like curves filling a world-tube $\Omega$. Let the hypersurfaces $S$ - with local equation $f\left(x^{1}, x^{2}, x^{3}, x^{4}\right)=0$ - and $B$ - with local equation $x^{4}=0$ be the boundary of $\Omega$ and a spatial section of $\Omega$ respectively.

Finally, let $V^{i}$ denote the field of unit tangents to the lines of $\Gamma$. To the field $V^{i}$ we associate two projection operators $\mathscr{P}$ and $\mathscr{N}$, acting on the vector bundle of $\Omega$, and mapping every vector field $A^{i}$ into the fields

$$
\begin{aligned}
& \mathscr{P}\left(A^{i}\right)=-V^{i} V_{j} A^{j}, \\
& \mathscr{N}\left(A^{i}\right)=\left(\delta_{j}^{i}+V^{i} V_{j}\right) A^{j}
\end{aligned}
$$

respectively tangent and normal to the lines of $\Gamma$.

By taking tensor products of copies of $\mathscr{P}$ and $\mathscr{N}$, we can induce projection operators $\mathscr{P} \otimes \cdots \otimes \mathscr{N} \otimes \cdots$ on every tensor bundle of $\Omega$ :

$$
\mathscr{P} \otimes \cdots \otimes \mathscr{N} \otimes \cdots\left(W^{i_{1} \ldots i_{r}}\right)=\left(-V^{i_{1}} V_{j_{1}}\right) \ldots\left(\delta_{j_{k}}^{i_{k}}+V^{i_{k}} V_{j_{k}}\right) \ldots W^{j_{1} \ldots j_{r}} .
$$

${ }^{2}$ The notation $\tilde{C}_{n}$ stands for "piecewise $C_{n}$ "; the notation $\left(C_{m}, \tilde{C}_{n}\right)(m<n)$ for " $C_{m}$, piecewise $C_{n} "$. 
In particular, in the space of symmetric tensors of rank 2, we have the obvious relation

$$
\mathscr{P} \otimes \mathscr{N}\left(W^{i j}\right)=\overline{\mathscr{N} \otimes \mathscr{P}\left(W^{i j}\right)}
$$

(the symbol $\sim$ denoting interchange of the indices), so that we have only three independent projections: $\mathscr{P} \otimes \mathscr{P}, \mathscr{N} \otimes \mathscr{N}$ and

$$
\mathscr{P} \odot \mathscr{N} \stackrel{\text { def. }}{=} \mathscr{P} \otimes \mathscr{N}+\mathscr{N} \otimes \mathscr{P} .
$$

In the domain $\Omega$ now consider the system

$$
G_{i j}=-k T_{i j}
$$

together with a suitable set of initial and boundary data specified on $B$ and on $S$ respectively.

Consider also the following systems

$$
\begin{aligned}
& (\alpha)\left\{\begin{array}{l}
\mathscr{N} \otimes \mathscr{N}\left[R^{i j}+k\left(T^{i j}-\frac{1}{2} T g^{i j}\right)\right]=0, \\
T^{i j}{ }_{\mu j}=0,
\end{array}\right. \\
& (\beta)\left\{\begin{array}{l}
\mathcal{N} \otimes \mathscr{N}\left[R^{i j}+k\left(T^{i j}-\frac{1}{2} T g^{i j}\right)\right]=0, \\
\mathscr{P} \odot \mathscr{N}\left(G^{i j}+k T^{i j}\right)=0, \\
\mathscr{P}\left(T^{i j}{ }^{\prime j}\right)=0,
\end{array}\right. \\
& \left(\text { () } \left\{\begin{array}{l}
\mathscr{N} \otimes \mathscr{N}\left[R^{i j}+k\left(T^{i j}-\frac{1}{2} T g^{i j}\right)\right]=0, \\
\mathscr{P} \otimes \mathscr{P}\left(G^{i j}+k T^{i j}\right)=0, \\
\mathscr{N}\left(T^{i j}{ }^{i j}\right)=0 .
\end{array}\right.\right.
\end{aligned}
$$

We shall prove that, in the initial and boundary value problem, the system (2.4) is mathematically equivalent to any of the systems $(\alpha),(\beta)$ and $(\gamma)$, provided that the data on $B$ and on $S$ satisfy the consistency conditions $[15 \div 18]$

$$
\begin{aligned}
& G_{i}^{4}=-k T_{i}^{4} \text { on } B, \\
& \left(G^{i j}+k T^{i j}\right) f_{, \jmath}=0 \text { on } S .
\end{aligned}
$$

In particular, as far as the equivalence between Eqs. (2.4) and the system $(\alpha)$ is concerned, our result reduces to Lichnerowicz's one $[15 \div 17]$ under the further restrictions

(i) the congruence $\Gamma$ is normal,

(ii) the degree of smothness of $\Gamma$ is not $\left(C_{1}, \tilde{C}_{2}\right)$, but $\left(C_{2}, \tilde{C}_{4}\right)$,

(iii) the problem is a pure Cauchy one (i.e. $\Omega=\mathscr{V}_{4}$ ).

In a previous paper [18] we have already shown how the restriction (iii) can be dropped. The fact that we can now drop the restrictions (i) and (ii) too, is particularly worthwhile in the discussion of the problem 
of motion, as it enables us to identify the congruence $\Gamma$ with the congruence of stream lines of an arbitrary material continuum.

In order to prove our result, we shall follow an abstract method [13], in which the tensor field $G_{i j}+k T_{i j}$ is replaced by an arbitrary symmetric tensor field $W_{i j}$. For every symmetric field $W_{i j}$, we define the conjugate field $W_{i j}^{*}$ by

$$
W_{i j}^{*}=W_{i j}-\frac{1}{2} W g_{i j} ; \quad W=g^{a b} W_{a b} .
$$

Eq. (2.7) implies

$$
\left(W_{i j}^{*}\right)^{*}=W_{i j} .
$$

Lemma 2.1. Let $V^{i}$ be an arbitrary time-like unit vector field, and let $\mathcal{N}$ be the projection operator associated to $V^{i}$ by Eq. (2.2).

Then, the contravariant components $W^{i j}$ of any symmetric tensor field may always be expressed in the linear form

$$
W^{i j}=A_{p q}^{i j} \mathscr{N} \otimes \mathscr{N}\left(W^{* p q}\right)+B^{i j}{ }_{k} W^{k p} V_{p}
$$

the coefficients $A^{i j}{ }_{p q}$ and $B^{i j}{ }_{k}$ being functions of the metric and of $V^{i}$. In particular

$$
B^{i j}{ }_{k}=-\left(\delta_{k}^{i} V^{j}+V^{i} \delta_{k}^{j}-V_{k} g^{i j}\right) .
$$

Proof. By a proper choice of the local co-ordinates, we may always require

$$
V^{i}=\gamma^{i} \stackrel{\text { def. }}{=} \frac{\delta_{4}^{i}}{\sqrt{-g_{44}}} ; \quad V_{i}=\gamma_{i}=\frac{g_{i 4}}{\sqrt{-g_{44}}}
$$

at any given point of $\mathscr{V}_{4}$.

A straightforward application of Eq. (2.3) shows that Eq. (2.10) implies

$$
\mathscr{N} \otimes \mathscr{N}\left(W^{* p q}\right)=C^{p q}{ }_{\alpha \beta} W^{* \alpha \beta}
$$

the coefficients $C^{p q}{ }_{\alpha \beta}$ being functions of the metric. Moreover

$$
W^{k p} V_{p}=W^{k p} \gamma_{p}=\frac{W_{4}^{k}}{\sqrt{-g_{44}}} .
$$

Now, we have

$$
\begin{gathered}
W_{4}^{\alpha}=W_{4}^{* \alpha}=g_{4 \beta} W^{* \alpha \beta}+g_{44} W^{* \alpha 4} \\
W_{4}^{4}=W_{4}^{* 4}-\frac{1}{2} W^{*}=-\frac{1}{2}\left(g_{\alpha \beta} W^{* \alpha \beta}-g_{44} W^{* 44}\right)
\end{gathered}
$$

Eqs. $(2.13 \mathrm{a}, \mathrm{b})$ may be easily solved for $W^{* \alpha 4}$ and $W^{* 44}$.

Therefore, all the components $W^{* i j}$ may be expressed linearly in terms of $W^{* \alpha \beta}$ and $W_{4}^{k}$. By Eq. $\left(2.7^{\prime}\right)$, the same is then true of the components $W^{i j}$. 
By Eqs. (2.11), (2.12), this statement is equivalent to Eq. (2.8) in the local frame in which Eq. (2.10) holds. Finally, in view of the tensorial character of Eq. (2.8), the same conclusion is valid in every frame.

This proves the first part of Lemma 2.1. The proof of Eq. (2.9) may be obtained by direct computation.

By Lemma 2.1, every symmetric tensor field $W^{i j}$ satisfying

$$
\mathscr{N} \otimes \mathscr{N}\left(W^{* i j}\right)=0
$$

is necessarily of the form

with $\mu^{k}=W^{k l} V_{l}$.

$$
W^{i j}=B^{i j}{ }_{k} \mu^{k}
$$

Eqs. (2.9), (2.14) and our previous definitions imply the following algebraic relations:

$$
\begin{aligned}
& \mathscr{P} \odot \mathscr{N}\left(W^{i j}\right)=0 \quad \text { iff } \quad \mathscr{N}\left(\mu^{j}\right)=0, \\
& \mathscr{P} \otimes \mathscr{P}\left(W^{i j}\right)=0 \quad \text { iff } \quad \mathscr{P}\left(\mu^{j}\right)=0, \\
& W_{i}^{4}=0 \quad \text { iff } \quad \mu^{j}=0\left(\Rightarrow W^{i j}=0\right) .
\end{aligned}
$$

Let us now go back to the world-tube $\Omega$ and to the vector field $V^{i}$ introduced at the beginning of this section.

In the domain $\Omega$, we consider a symmetric tensor field $W^{i j}$ of the form (2.14) satisfying

$$
\begin{array}{rll}
W_{i}^{4}=0 & \text { on } & B, \\
W^{i j} f_{{ }_{j}}=0 & \text { on } & S .
\end{array}
$$

Lemma 2.2. Let the symmetric tensor field $W^{i j} \in C_{1}(\Omega)$ satisfy Eqs. (2.14), (2.18), (2.19). Then, in the domain $\Omega$, the following statements are mathematically equivalent :

(I) $W^{i j}=0$,

(II) $W^{i j}=0$,

(III) $\left\{\begin{array}{l}\mathscr{P} \odot \mathscr{N}\left(W^{i j}\right)=0, \\ \mathscr{P}\left(W^{i j}{ }_{\| j}\right)=0,\end{array}\right.$

(IV) $\left\{\begin{array}{l}\mathscr{P} \otimes \mathscr{P}\left(W^{i j}\right)=0 \\ \mathscr{N}\left(W^{i j}{ }^{\prime}\right)=0\end{array}\right.$

Proof. Obviously, the statement (I) implies all the others. The relevant part of the proof is to show that (II) $\Rightarrow(\mathrm{I}),(\mathrm{III}) \Rightarrow(\mathrm{I})$ and $(\mathrm{IV}) \Rightarrow(\mathrm{I})$.

(i) Assume (II). Eq. (II) is mathematically equivalent to

$$
\left(g_{i p}+2 V_{i} V_{p}\right) W_{1 / j}^{p j}=0
$$


as the matrix $g_{i p}+2 V_{i} V_{p}$ is non-singular for any time-like unit vector field $V^{i}$ (check). If we evaluate the covariant derivatives explicitly, and make use of Eqs. (2.9), (2.14), we can write Eq. (2.20) in the form

$$
M_{i}{ }_{k} \mu^{k}{ }_{j}+N_{i k} \mu^{k}=0
$$

where

$$
M_{i k}{ }_{k}=B_{i k}{ }^{j}+2 V_{i} V_{p} B^{p j}{ }_{k}=g_{i k} V^{j}-V_{i} \delta_{k}{ }^{j}-V_{k} \delta_{i}{ }^{j}
$$

and the coefficients $N_{i k}$ are functions of $g_{p q}, q_{p q, r}, V_{p}, V_{p, r}$.

Ve may regard Eqs. (2.21) as a system of partial differential equations for the unknowns $\mu^{k}$. Eqs. (2.18), (2.19) provide the initial data

$$
\mu^{k}=0 \quad \text { on } B
$$

(by Eq. (2.17)), and the boundary conditions

$$
B^{i j}{ }_{k} f, \mu_{j} \mu^{k}=M_{i}{ }_{k} f_{j}, \mu_{j} \mu^{k}=0 \text { on } S .
$$

We shall now prove that the system (2.21), (2.23), (2.24) admits the unique solution $\mu^{k}=0$.

To this purpose we notice that Eqs. (2.22) imply $M_{i}{ }^{j}=M_{k}{ }^{j}$.

Moreover, for any time-like hypersurface $\varphi\left(x^{1} x^{2} x^{3} x^{4}\right)=0$ satisfying $\varphi_{, 4}>0$, the matrix $\Phi_{i k}=M_{i k}{ }^{j} \varphi_{, j}$ is positive definite. In fact, by a proper choice of the local coordinates, we may always require $V^{i}=\gamma^{i}$ and $\varphi,_{i}=a \delta_{i}^{4}(a>0)$, at any given point $x \in \Omega$. In these co-ordinates we have, by Eq. (2.22)

$\Phi_{i k} \lambda^{i} \lambda^{k}=\frac{a}{\sqrt{-g_{44}}}\left(g_{i k}-2 g_{i 4} \delta_{k}^{4}\right) \lambda^{i} \lambda^{k}=\frac{a}{\sqrt{-g_{44}}}\left(g_{\alpha \beta} \lambda^{\alpha} \lambda^{\beta}-g_{44} \lambda^{4} \lambda^{4}\right)>0$

unless $\hat{i}^{i} \equiv 0$.

Therefore, by Sylvester's law, the matrix $\Phi_{i k}$ is positive definite in every frame of reference.

The previous properties show that the system (2.21) is symmetric and hyperbolic (see Appendix). Also, recalling that the vector field $V^{i}$ is $\left(C, \tilde{C}_{1}\right)$ (by the prescribed properties of the congruence $\Gamma$ ), while the metric tensor $g_{i j}$ is $\left(C_{1}, \tilde{C}_{3}\right)$, we see that the coefficients $M_{i}{ }^{j}{ }_{k}$ and $N_{i k}$ are respectively $\left(C, \tilde{C}_{1}\right)$ and $\tilde{C}$.

We can therefore apply the general uniqueness theorem stated in the Appendix.

Taking Eqs. (2.23), (2.24) into account, we obtain $\mu^{k}=0$ in $\Omega$, and therefore, by Eq. (2.14), $W^{i j}=0$ in $\Omega$.

This completes the proof that (II) $\Rightarrow$ (I).

(ii) Assume (III). Then, by Eq. (2.15) and the first condition in (III)

$$
\mu^{j}=c V^{j}
$$


i.e., by Eqs. (2.9), (2.14)

$$
W^{i j}=-c\left(2 V^{i} V^{j}+g^{i j}\right) .
$$

Eq. (2.26) and the second condition in (III) imply

$$
V^{j} c,_{j}+2 c V_{/ / j}^{j}=0 \text {. }
$$

Moreover, by Eqs. (2.18), (2.19), (2.26), taking Eq. (2.17) into account

$$
\begin{gathered}
c=0 \text { on } B, \\
V_{i}\left(W^{i j} f,{ }_{j}\right)=c V^{j} f,{ }_{j}=0 \text { on } S .
\end{gathered}
$$

Finally, by the time-like character of the vector field $V^{i}$, we have

$$
V^{j} \varphi,{ }_{j}>0
$$

for any time-like hypersurface $\varphi\left(x^{1} x^{2} x^{3} x^{4}\right)=0$ satisfying $\varphi,{ }_{4}>0$.

Using the condition $V^{i} \in\left(C, \hat{C}_{1}\right)$, we see that we can again apply the uniqueness theorem stated in the Appendix. This gives $c=0$ in $\Omega$, i.e., by Eq. (2.26), $W^{i j}=0$ in $\Omega$. Therefore also (III) $\Rightarrow$ (I).

(iii) Assume (IV). Then, by Eq. (2.16) and the first condition in (IV)

i.e., by Eqs. (2.9), (2.14)

$$
\mu^{k} V_{k}=0
$$

$$
W^{i j}=-\left(\delta_{k}^{i} V^{j}+V^{i} \delta_{k}^{j}\right) \mu^{k},
$$

Eq. (2.31) and the second condition in (IV) imply

$$
-\left(g_{i p}+V_{i} V_{p}\right) W_{/ / j}^{p j}=\left(g_{i k}+V_{i} V_{k}\right) V^{j} \mu^{k}{ }_{/ j}+H_{i k} \mu^{k}=0
$$

the coefficients $H_{i k}$ being function of $g_{p q}, g_{p q, r}, V_{i}, V_{i, r}$.

If we now differentiate Eq. (2.30) with respect to $x^{j}$, we see that Eq. (2.32) may be written in the equivalent form

with

$$
\left(g_{i k}+2 V_{i} V_{k}\right) V^{j} \mu^{k},_{j}+\tilde{H}_{i k} \mu^{k}=0
$$

$$
\tilde{H}_{i k}=H_{i k}+V_{i} V^{j} V_{k_{/ /}}+\left(g_{i p}+2 V_{i} V_{p}\right) V^{i}\left\{\begin{array}{c}
{ }_{k} \\
j
\end{array}\right\}
$$

The system (2.33) is again symmetric and hyperbolic, as may be easily checked.

Moreover, Eqs. (2.17), (2.18), (2.19), (2.30), (2.31) imply the initial data

$$
\mu^{j}=0 \text { on } B
$$

and the boundary conditions

$$
\left(\delta_{p}^{i}+V^{i} V_{p}\right) W^{p j} f_{{ }_{j}}=\mu^{i} V^{j} f_{, j}=0 \quad \text { on } S .
$$


Finally, the coefficients of the system (2.33) satisfy the continuity requirements stated in the Appendix. Therefore, by the uniqueness theorem, we deduce again $\mu^{k}=0$ in $\Omega$, i.e., by Eq. $(2.31), W^{i j}=0$ in $\Omega$. Thus, also (IV) $\Rightarrow(\mathrm{I})$, and the proof of Lemma 2.2 is complete.

We now consider the tensor field (defined in the domain $\Omega$ )

$$
W^{i j}=G^{i j}+k T^{i j} \text {. }
$$

Then, by the topological structure of $\mathscr{V}_{4}$, Eqs. (2.18), (2.19), are identically satisfied, and are in fact to be regarded as consistency conditions for the initial and boundary data of the gravitational problem [18]. Also, by Lemma 2.1. Eq. (2.14) is mathematically equivalent to

$$
\mathscr{N} \otimes \mathscr{N}\left(W^{* i j}\right)=\mathscr{N} \otimes \mathscr{N}\left[R^{i j}+k\left(T^{i j}-\frac{1}{2} T g^{i j}\right)\right]=0 .
$$

Finally, in view of the Bianchi identities, the equation $W^{i j}{ }_{/ j}=0$ can now be replaced by $T^{i j}{ }_{1 / 3}=0$.

If we collect all these facts, and apply Lemma 2.2, we obtain the result stated at the beginning of this section, namely: the gravitational Eqs. (2.4) are mathematically equivalent to any of the systems $(\alpha),(\beta)$ and $(\gamma)$, provided that the initial and boundary data satisfy the consistency conditions (2.5), (2.6).

\section{$\S 3$. The Problem of Motion}

Let $T_{i j}$ be the energy-momentum tensor of a material continuum whose hystory is contained in the world-tube $\Omega$ introduced in Section 2.

Consider the eigenvalue equation

$$
\left(T_{i j}+\mu g_{i j}\right) V^{j}=0
$$

with the normalization

$$
g_{i j} V^{i} V^{j}=-1 .
$$

We assume that the eigenvalue $\mu$ exists, and is real, positive and non-degenerate.

In this case, the tensor $T_{i j}$ admits a unique decomposition of the form

with

$$
T_{i j}=\mu V_{i} V_{j}-S_{i j}
$$

$$
S_{i j} V^{j}=0 .
$$

We assume $V^{i} \in\left(C, \tilde{C}_{1}\right)$, and identify the quantities $\mu, V^{i}$ and $S_{i j}$ with the density, four-velocity and stress tensor of the continuum respectively.

The problem of motion consists of the determination of the density $\mu$ and of the congruence $\Gamma$ of stream-lines of the continuum, these being world-lines having $V^{i}$ for unit tangents. 
To achieve this goal we have at our disposal:

(i) Einstein's gravitational equations

$$
G_{i j}=-k T_{i j},
$$

(ii) the state equations describing the physical structure of the continuum,

(iii) a suitable set of initial and boundary data.

We assume that the geometry of space-time inside $\Omega$ is uniquely determined by (i), (ii), and (iii)

Under these conditions, the problem of motion is well posed and physically acceptable. Our aim is to discuss its general structure.

To this purpose, we consider the projection operators $\mathscr{P}$ and $\mathscr{N}$ associated to the vector field $V^{i}$ by Eqs. (2.1), (2.2). In terms of these operators, Eqs. (3.1) are mathematically equivalent to

$$
\begin{aligned}
\mathscr{P} \odot \mathscr{N}\left(T^{i j}\right) & =0, \\
\mathscr{P} \otimes \mathscr{P}\left(T^{i j}\right) & =\mu V^{i} V^{j}
\end{aligned}
$$

while Eqs. (3.3), (3.4) imply

$$
\mathscr{N} \otimes \mathscr{N}\left(T^{i j}\right)=-S^{i j} .
$$

One can easily check that Eqs. (3.6) are satisfied if anf only if the timelike vector field $V^{i}$ is an eigenvector of $T_{i j}$, irrespective of the value of $\mu$.

Therefore, Eqs. (3.6) define the four-velocity $V^{2}$ in terms of $T_{i j}$, and Eqs. (3.7), (3.8) define the density $\mu$ and the stress tensor $S_{i j}$ in terms of $V^{i}$ and $T_{i j}$.

If we now use Eqs. $(3.6) \div(3.8)$ and Lemma 2.1, we can express the field equations (3.5) in the form

$$
\begin{gathered}
\mathscr{P} \odot \mathscr{N}\left(G^{i j}\right)=0, \\
\mathscr{P} \otimes \mathscr{P}\left(G^{i j}\right)=-k V^{i} V^{j}, \\
\mathscr{N} \otimes \mathscr{N}\left[R^{i j}+k\left(T^{i j}-\frac{1}{2} T g^{i j}\right)\right]=0 .
\end{gathered}
$$

Using the same argument as before, we see that Eqs. (3.9), (3.10) determine $V^{i}$ and $\mu$ in terms of the gravitational fields $g^{i j}$.

In this sense, Eqs. (3.9), (3.10) are mathematically equivalent to the equations of motion for the given continuum, as they express the relevant kinematical quantities in terms of the dynamical effects.

The reason why we do not assert that Eqs. (3.9), (3.10) are the equations of motion is based on the fact that the equations of motion should in principle be able to determine the evolution of an arbitrary infinitesimal 
portion $\delta$ of the continuum in terms of the dynamical effects generated by the rest of the continuum and by the external sources.

Now, let $\Omega^{\prime} \subset \Omega$ be the (infinitesimal) world-tube containing the hystory of $\delta$.

Then Eqs. (3.9), (3.10) express $\mu$ and $V^{i}$ inside $\Omega^{\prime}$ in terms of the Einstein tensor $G_{i j}$ inside $\Omega^{\prime}$, i.e. in terms of the dynamical effects generated by $\delta$ itself.

In particular, if we neglect these effects by letting $k \rightarrow 0$ (i.e. $G_{i j} \rightarrow 0$ ) in $\Omega^{\prime}$, Eqs. (3.9), (3.10) become identities, and do not determine the evolution of $\delta$ any longer.

Thus we conclude

Theorem 3.1. The equations of motion for a material continuum of the form (3.3) are completely characterized by the following properties:

a) they must be mathematically equivalent to Eqs. (3.9), (3.10);

b) they must remain non-trivial (i.e., they must not reduce to identities) in the limit $k \rightarrow 0$.

If we now apply to the system $(3.9) \div(3.11)$ the results of Section 2 , taking Eqs. (3.6), (3.7) into account, we see that Eq. (3.9) may be replaced by

$$
\mathscr{N}\left(T^{i j}{ }_{/ /}\right)=0,
$$

while Eq. (3.10) may be replaced by

$$
\mathscr{P}\left(T^{i j}{ }_{/ /,}\right)=0 .
$$

Eqs. (3.11), (3.12) are mathematically equivalent to the divergence equations

$$
T^{i j}=0 \text {. }
$$

These equations are surely non trivial in the limit $k \rightarrow 0$, as they are perfectly meaningful even in a flat space-time $\left(k=0, G_{i j}=0\right)$.

Therefore, in view of Theorem 3.1, we conclude.

Corollary 3.1. The equations of motion for a material continuum of the form (3.3) are the divergence equations (3.13). More precisely, if we split these equations into the system (3.11), (3.12), Eqs. (3.11) determine the evolution of the four-velocity field $V^{i}$ (and therefore the congruence of stream-lines of the material continuum), while Eq. (3.12) determines the evolution of the density.

The proof of all these assertions follows easily from the previous arguments, and will be omitted.

This work has been performed while the Author was a scholar at the Dublin Institute for Advanced Studies, School of Theoretical Physics.

The Author wishes to express his sincere gratitude to Prof. J. L. Synge and Prof. J. R. McConnell for their warm hospitality. 


\section{Appendix: Uniqueness Theorem for Symmetric Hyperbolic Systems of Partial Differential Equations ${ }^{3}$}

In the space-time manifold $\mathscr{V}_{4}$, consider the linear operator

$$
L=A^{i} \frac{\partial}{\partial x^{i}}+B
$$

where $A^{i}$ and $B$ are real $n \times n$ matrices whose entries are functions of $\left(x^{1} x^{2} x^{3} x^{4}\right)$.

We assume:

(i) all the matrices $A^{i}$ are symmetric,

(ii) the matrix $A^{i} \varphi,_{i}$ is positive definite for any time-like hypersurface $\varphi\left(x^{1}, x^{2}, x^{3}, x^{4}\right)=0$ satisfying $\varphi,_{4}>0$ (so that, in particular, $A^{4}$ is positive definite).

(iii) $A^{i} \in\left(C, \tilde{C}_{1}\right)$ and $B \in \tilde{C}$.

The first two conditions imply that $L$ is a symmetric hyperbolic differential operator.

Now, let $\Omega$ be the domain introduced in Section 2. In $\Omega$ consider the field $u=\left(u^{1}, \ldots, u^{n}\right)$, where the components $u^{k}$ are $C_{1}$ functions of the space-time co-ordinates.

We shall prove the following

Theorem A.1. The system

$$
L[u]=A^{i} u_{i}+B u=0
$$

admits the unique solution $u=0$ in $\Omega$, provided that the following conditions are satisfied:

(I) $u=0$ on $B$,

(II) $\left(u \mid A^{i} f,{ }_{i} u\right) \geqq 0 \quad$ on $S .{ }^{4}$

Proof. By setting $u=e^{\alpha x^{4}}$. v, we can write the system (A.2) in the equivalent form

$$
\tilde{L}[v]=A^{i} v,_{i}+\left(B+\alpha A^{4}\right) v=0 .
$$

Using Eq. (A.3) and the condition (i) we obtain

$$
2(v \mid \tilde{L}[v])=2\left(v \mid A^{i} v,_{i}\right)+2\left(v \mid\left[B+\alpha A^{4}\right] v\right)=\left(v \mid A^{i} v\right)_{i}+(v \mid \tilde{B} v)=0
$$

with

$$
\tilde{B}=2\left(B+\alpha A^{4}\right)-A^{i},{ }_{i} .
$$

3 The material of this Appendix is a straightforward generalization of the results shown in Ref. [14]; it is reported here for convenience of the reader. The notation, terminology, etc., are the same as in [14].

4 The symbol (|) is used here to denote the usual scalar product in the Hilbert space $\mathbb{R}^{n}$, namely $(u \mid v)=u^{1} v^{1}+\cdots+u^{n} v^{n}$. 
By the conditions (ii), (iii), and the arbitrariness of $\alpha$, we see that it is always possible to require that the matrix $\tilde{B}$ be positive definite in the whole domain $\Omega$.

Now, let $\Sigma$ be any time-like section of $\Omega$, with local equation $\varphi\left(x^{1}, x^{2}, x^{3}, x^{4}\right)=0, \varphi,{ }_{4}>0$.

We integrate Eq. (A.4) in the domain $\mathscr{D}$ bounded by the hypersurfaces $B, S$ and $\Sigma$, and use Gauss theorem. This gives

$$
-\int_{B}\left(v \mid A^{4} v\right)+\int_{S}\left(v \mid A^{j} v\right) f_{j}+\int_{\Sigma}\left(v \mid A^{j} v\right) \varphi,{ }_{j}+\iint_{\mathscr{D}}(v \mid \tilde{B} v)=0 .
$$

The first term in Eq. (A.5) vanishes in view of the condition (I). Moreover, by the conditions (II), (ii) and our previous arguments, all the other terms in Eq. (A.5) are necessarily non-negative.

Therefore, in order that Eq. (A.5) be satisfied, we must necessarily have $v=0$ in $\mathscr{D}$. By the arbitrariness of $\Sigma$, this implies $v=0$ in $\Omega$, i.e. $u=e^{\alpha x^{4}} v=0$ in $\Omega$.

\section{References}

1. Einstein, A., Grommer, J.: S. B. Berlin. Akad. Wiss. p. 2 (1927).

2. - Infeld, L., Hoffmann, B.: Ann. Math. 39, 65 (1938).

3. - - Ann. Math. 41, 455 (1940).

4. - - Canad. J. Math. 1, 209 (1949).

5. Infeld, L., Schild, A.: Rev. Mod. Phys. 21, 408 (1949).

6. - Acta Phys. Pol. 13, 187 (1954).

7. - Plebansky, J.: Motion and Relativity. Warszawa: Pergamon Press 1960.

8. Fock, V. A.: J. Phys. Moscow 1, 81 (1939).

9. - Theory of Space, Time and Gravitation. Moscow: Pergamon Press 1964.

10. - Rev. Mod. Phys. 29, 325 (1957).

11. Bazanski, S.: The problem of motion. In: Recent Developments in General Relativity. Warszawa: Pergamon Press/ Polish Sci. Publ. 1962.

12. - The equations of motion and the action principle in General Relativity. Warszawa: Pergamon Press/Polish Sci. Publ. 1962.

13. Synge, J. L.: Relativity: the General Theory. Amsterdam: North Holland 1960.

14. Courant, R., Hilbert, D.: Methods of Mathematical Physics, Vol. II. New York: Interscience 1962.

15. Lichnerowicz, A.: Théories relativistes de la gravitation et de l'électromagnetisme. Paris: Masson 1955.

16. Fourés Bruhat, Y.: The Cauchy problem. In: Gravitation: An introduction to current research. New York: Witten 1962.

17. Lichnerowicz, A., Fourés Bruhat, Y.: Problemes mathématiques en Relativité. In: Recent developments in General Relativity. Warszawa: Pergamon Press/Polish Sci. Publ. 1962.

18. Massa, E.: Commun. math. Phys. 12, 246 (1969).

Enrico Massa

Istituto di Matematica

Università di Genova

Via L.B. Alberti, 4

I-16132 Genova, Italia 